\title{
Evaluation of Bowel Habits in Patients with Acne Vulgaris
}

\author{
Munise Daye ${ }^{1}$, Fatma Cihan ${ }^{1}$, Begüm Işık ${ }^{1}$, and Berna Hafızoğlu ${ }^{1}$ \\ ${ }^{1}$ Necmettin Erbakan University Meram Medical Faculty Hospital
}

February 5, 2021

\begin{abstract}
Purpose: To evaluate the bowel habits of patients with acne vulgaris Method: In this case control study, socio-demographic characteristics of the participants (age, gender, marital status, educational status, profession, economic status, smoking-alcohol habits, chronic diseases, previous operations, people they live with, diet) were questioned and the global acne grading scores of the patients were calculated. Bristol Stool Scale, Rome III Criteria for IBS, International Physical Activity Questionnaire-Short Form, Hospital Anxiety Depression and KADF (dietary fiber information) scales fulfilled. Results: The patient group consisted 102 and the control group consisted 104 participants. The mean age was $20.9 \pm 3.9$ years old in the case group and $21.8 \pm$ 5.0 years old in the control group. Patient and control groups were similar in terms of age and gender. Smoking rates were significantly higher in the patient group $(\mathrm{p}=0,035)$. The amount of coffee consumed per day were significantly higher in the patient group $(\mathrm{p}=0,040)$. According to the global acne grading scores, 55.9\% ( $\mathrm{n}=57)$ had medium, 39.2\% ( $\mathrm{n}=40)$ had mild, $3.9 \%(\mathrm{n}=4)$ had severe and $1 \%(\mathrm{n}=1)$ had very severe acne. Anxiety scores were found to be significantly higher in the patient group $(\mathrm{p}=0.005)$. When the case and control groups were compared for IBS presence, no significant difference was found ( $\mathrm{p}$ $=0.317)$. Also, IBS was not related with acne severity $(\mathrm{p}=0.162)$. Conclusion: Further large sample sized studies are needed on this subject, as there is strong evidence about brain-gut-skin axis existence.
\end{abstract}

\section{INTRODUCTION}

Acne vulgaris is a chronic inflammatory disease of the pilosebaceous unit and is characterized by seborrhea, open/closed comedones, papules, pustules and nodulocystic lesions(1). The cause of acne vulgaris increased sebaceous gland activity, abnormal follicular hyper keratinization, microbial hyper-colonization of the follicular canal and increased inflammation(2). Lifestyle, diet, testosterone levels, gastrointestinal dysfunction, bacterial infection, and psychological factors play a role in the pathogenesis and development of acne. Exercise, even moderate activity such as walking, can help to treat both constipation and acne(3). Another disease affecting the brain and intestinal axis by inflammatory process is irritable bowel syndrome. Irritable bowel syndrome (IBS) is the most commonly diagnosed disease without an underlying organic pathology, characterized by the presence of abdominal pain or discomfort with variable bowel habits (4). Its prevalence is generally between $12-30 \%$ (5). IBS symptoms are identified in four subtypes according to Rome III criteria. Bristol Stool Scale (25) provides information about the consistency, thickness and amount of stool. Studies reveal that IBS is more common in women than men (6). IBS has been considered a brain-intestinal disease. In susceptible individuals (for example, those with a genetic predisposition or exposed to environmental factors), the association with psychological stress (eg anxiety, depression or somatization) and an infectious or inflammatory response can alter intestinal permeability, contributing to the onset of IBS symptoms (7). Depression and somatization can also lead to the development of IBS symptoms by mediating changes in intestinal permeability, immune system, and microbiome (7). Localized inflammation develops as the intestinal permeability increases, and then the number of inflammatory cells (histamine, tryptase, serotonin, TNF-, proteases and interleukins) increases (7). Although there are many data on acne and microbiota in the literature, data on acne and intestinal habits have not been determined. Normal functioning of gastrointestinal tract can influence benefit from oral treatment besides adverse events (7). The aim of this study 
is to evaluate the impact of bowel habits, IBS, physical activity, and dietary fiber intake in acne vulgaris patients.

\section{MATERIAL METHOD}

The ethics committee of our faculty approved this case control study. Dermatology patients with acne complaints (over 18 years old, volunteered to participate) were included through one month period. Age and gender matched healthy relatives of the patients who applied to Dermatology and Family Medicine Outpatient Clinics without acne and accompanying dermatosis were included as the control group. The exclusion criteria were: refusal to participate the study, being under 18 years old and over 65 years old, mental retardation, verbal communication defects, people whose native language was not Turkish, diagnosed gastrointestinal disease, previous gastrointestinal surgery, diabetes mellitus, thyroid diseases, Addison's disease, presence of diseases known to alter gastrointestinal functions, pregnancy, using drugs that affect intestinal habits such as iron preparations and antacids 3 weeks prior to the study.

In the first part of the questionnaire, the demographic characteristics of the participants (age, gender, marital status, educational status, profession, economic status, smoking-alcohol habits, diagnosed diseases, previous operations, people they live with, diet) were questioned and the global acne score was calculated. In the second part, Bristol Stool Scale, Rome III Criteria, International Physical Activity Questionnaire-Short Form, Hospital Anxiety Depression, KADF (dietary fiber information) scalesfulfilled.

The global acne grading system (GAGS) is used to determine the clinical severity of acne. The upper part of the face, chest and back region is divided into six parts, taking into account the width of the region and the density and distribution of the pilosebaceous unit in that region, a coefficient for each region are (forehead, right cheek and left cheek $=2$, nose and chin $=1$, chest and upper back $=3$ ) given. Acne lesions were also graded between $0-4$ according to their types (no lesions $=0,1$ comedone $=1,1$ papule $=2$, [?]1 pustules $=3$, [?]1 nodules $=4)$. Each region is evaluated separately, the score obtained according to the most severe lesion type in that region is multiplied by the coefficient of that region, and a score is determined for each region, and the global acne score is calculated with the sum of the scores of the six regions. The total score ranges from 0 to 44 , acne severity is determined according to the global acne score. ( 0 point $=$ no acne, $1-18$ points $=$ mild acne, $19-30$ points $=$ moderate acne, $31-38$ points $=$ severe acne, $>39$ points $=$ very severe acne) (8).

HAD (Hospital Anxiety-Depression) Scale:

It was developed and validated by Zigmond and Snaith in 1983(32). It includes anxiety and depression subscales. It consists of 14 items, seven of them are depression (2., 4., 6., 8., 10., 12., 14) and seven are anxiety (1., 3., 5., 7., 9., 11., 13.) screening. Answers are in Likert format and scored between zero to three. Questions 2,4,7,9,12 and 14 are scored as $0,1,2,3$ points and $1,3,5,6,8,10,11$ and 13 . questions are scored as $3,2,1,0$ points. The scores from both subscales may vary between $0-21$ points. The Turkish validity and reliability study of HADS has been conducted by Aydemir et al (33). Cut-off scores of the Turkish version of the HADS scale are 10 points for anxiety subscale and 7 points for depression subscale(9).

KADF (Knowledge about dietary fiber) Scale:

Guine et al developed it in 2016. Its validity and reliability were tested by multicenter studies including Turkey(10). Awareness about Foods with Dietary Fiber is evaluated with 6 questions, the Relationship between Dietary Fiber and Various Foods with 6 questions and the Relationship between Dietary Fiber and Diseases with 10 questions. The questions 3.4, 3.5, 5.2, 5.3, 5.4 and 5.6 are evaluated in reverse. The higher scores indicate the higher the level of knowledge about food fibers (10).

\section{Rome III Criteria:}

The Rome III criteria was published in 2006 and Turkish validity and reliability of was performed by Uran et al in 2012(11). Presence of at least 2 or more of the following along with recurrent abdominal pain or 
discomfort that exists for at least 3 days each month for the last 3 months, provided that it began at least 6 months before diagnosis(12).

1. Defecation recovery

2. Beginning with a change in the frequency of defecation

3. Beginning with a change in the form of gaita

* Supportive symptoms (not required for diagnosis)

1. Abnormality in the frequency of defecation

2. Anomaly in the form of gaita

3. Abnormal feces passage (feeling of strain, urgency or complete emptying)

4. Mucus outflow from the rectum

5. Feeling bloated (12).

International Physical Activity Questionnaire-Short Form (IPAQ-SF):

It was developed by the International Consensus group in Geneva in 1998 to evaluate the physical activity levels of individuals in international standards. Then, in 2000, it was accepted as a valid and reliable scale in 12 different countries. Sağlam et al. found in their study that both the short and long forms of IPAQ were valid and reliable in Turkish. This questionnaire evaluates the physical activity levels of individuals in the last seven days in four parts such as vigorous activities, moderate activities, walking and sitting. When calculating the total score, when the metabolic equation (MET) values given to the activities (intensive activity $=8 \mathrm{MET}$, moderate activity $=4 \mathrm{MET}$, walking $=3.3 \mathrm{MET})$ are multiplied by the duration of the activities (min) and the frequency of doing (number of days), the weekly MET- min scores are obtained. Physical activity levels of the individuals are divided into three categories as "inactive", "minimally active" and "very active" according to the scores obtained(13).

Statistical analysis

Number, percentage, mean and standard deviations were used to evaluate descriptive statistics. Shapiro Wilk and Kolmogorov Smirnov tests were used to check the compatibility of the data for normal distribution. Chi-square test for statistical analysis of categorical data, for statistical analysis of quantitative data, an independent $t$ test in paired groups, One Way ANOVA test (post hoc Tukey test) in groups with three or more, Mann Whitney U and Kruskall Wall tests are used. Statistical significance of the difference was accepted as $\mathrm{p}<0.05$. Correlation between parameters are made with Pearson correlation analysis. Correlation coefficient (r); Between 0.000-0.249 will be considered as weak relationship, between 0.250-0.499 as medium, between 0.500-0.749 as strong, between 0.750-1.000 as very strong relationship.

\section{RESULTS}

The patient group consisted of 102 and the control group consisted of 104 participants. The mean age was $20.9 \pm 3.9$ years old in the case group and $21.8 \pm 5.0$ years old in the control group. There was no significant difference between the groups in terms of age and gender $(\mathrm{p}>0.05)$. While the mean body mass index of the cases was $21.69 \pm 2.99 \mathrm{~kg} / \mathrm{m}^{2}$, it was $21.99 \pm 3.56 \mathrm{~kg} / \mathrm{m}^{2}$ in the control group $(\mathrm{p}=0.505)$. The mean acne duration was $3.86 \pm 2.57$ years. The socio-demographic characteristics of the participants are shown in Table 1. Smoking rates were found to be significantly higher in the patient group.

The nutritional and bowel habits of the participants are shown in Table 2. Meal habits, daily water and tea intake and number of vegetables, fruits, fast-food and defecations were not different in acne and control group. The amount of coffee consumed per day were significantly higher in the patient group $(\mathrm{p}=0,04)$.

According to the acne severity, $55.9 \%(\mathrm{n}=57)$ were medium, $39.2 \%(\mathrm{n}=40)$ were mild, $3.9 \%(\mathrm{n}=4)$ were severe and $1 \%$. $(\mathrm{n}=1)$ were found to have a very serious acne type. The mean global acne score was 20.32 
\pm 6.83 points. Anxiety-depression scores and dietary fiber knowledge scores of the study participants are shown in Table 3. Anxiety scores were found to be significantly higher in the patient group $(\mathrm{p}=0.005)$

Of the case group, $2.9 \%(\mathrm{n}=3)$ stated that their bowel pattern was changed during the period of acne. When the case group was classified according to Bristol stool scale, $33.3 \%(\mathrm{n}=34)$ were type 3, 27.5\% (n $=28)$ were type $2,12.7 \%(\mathrm{n}=13)$ were type $6,9.8 \%(\mathrm{n}=10)$ were type $5,7.8 \%(\mathrm{n}=8)$ were type $1,5.9 \%$ $(\mathrm{n}=6)$ were type 4 and $2.9 \%(\mathrm{n}=3)$ were type 7. In the control group, $37.5 \%(\mathrm{n}=39)$ were type $2,31.7 \%$ $(\mathrm{n}=33)$ were type $3,10.6 \%(\mathrm{n}=11)$ were type $1,10.6 \%(\mathrm{n}=11)$ were type $5,4.8 \%(\mathrm{n}=5)$ were type 4 and $4.8 \%(\mathrm{n}=5)$ were type 6 . There was no significant difference between the patient and control groups in terms of stool types $(\mathrm{p}=0.114)$.

Irritable Bowel Syndrome was present in $15.7 \%(\mathrm{n}=16)$ of the subjects participating in the study and $22.1 \%$ $(\mathrm{n}=23)$ of the control group. When the case and control groups were compared in terms of the presence of IBS, no significant difference was found between them $(\mathrm{p}=0.317)$.

When the cases were compared in terms of the presence of IBS according to acne severity, no significant relationship was found $(\mathrm{p}=0.162)$ (Table 4). Correlations between GAGS and Physical Activity Scores, BMI and dietary fiber knowledge of the subjects are demonstrated in Table 5.

\section{Discussion}

Acne is an important risk factor for psychological morbidity in young and advanced ages in both genders. It is related to satisfaction with appearance, decreased self-esteem and self-confidence, and increased internalization (anxiety and depression) and suicidal tendency. In a meta-analysis obtained from 42 studies on anxiety in acne cases, a significant relationship was found between acne and depression and anxiety and acne. It has been reported that the prevalence of depression and anxiety are higher in acne cases (14). According to the hypothesis of Stokes and Pillsbury; Emotional conditions such as depression and anxiety disrupt the function and microbiota of the gastrointestinal system, which paves the way for local and systemic inflammation. It is thought that there may be a serious relationship between the bacteriology and physiology of the gastrointestinal system, inflammatory skin diseases and mood. In addition, based on the evidence, $40 \%$ of acne cases have hypochlorhydria. This insufficient stomach acidity causes colon bacteria to migrate to the distal part of the small intestine and this disrupts the normal intestinal microflora. According to the authorities, microbial flora changes triggered by stress increase intestinal permeability and predispose to systemic and local inflammation $(2,3)$. Although the gut-brain skin axis hypothesis has been known for a long time, it is not well understood, but microbiome studies are opening the uncertainties here. In addition, the increase in Substance P secretion with the stimulation of the nerves that release substance P was observed in both acne cases and intestinal dysbiosis. Substance $\mathrm{P}$ is responsible for the release of proinflammatory cytokines in acne pathogenesis $(15,16,17,18)$.

Multiple comorbidities associated with IBS have been reported as somatic pain syndromes (fibromyalgia, chronic fatigue syndrome, and chronic pelvic pain), other gastrointestinal diseases (gastroesophageal reflux dyspepsia), and psychiatric diseases (major depression, anxiety, and somatization) (19). Low-grade inflammation was found to be important in IBS pathogenesis. In a study, serum inflammatory cytokines were found to be increased in IBS cases compared to controls (20,21). Increase in small intestine bacterial flora is associated with anxiety and depression, and recovery of flora provides emotional improvement) $(22,23)$. In half of the cases, IBS originates from the intestine first and psychological stress develops later $(4,5,6,7)$. Parallel to the literature a significant difference was found between the anxiety scores of the acne cases and the control group in this study, but there was no relationship with depression. It can be due to the number of cases or the characteristics of the selected sample. Depressive patients may not have been detected due to their unwillingness to participate in this study. When looked in terms of intestinal habits and IBS, patients with acne and control group did not differ. There was no relationship between the global acne scores and bowel habits or IBS. In the recent study by Demirbaş et al., 300 acne patients were evaluated with Rome IV criteria and IBS was found significantly higher in patients with acne. IBS was also correlated with the global acne scores (24). In our study, Rome III criteria were used because it was validated and reliable in 
Turkish (Ozgürsoy Uran.et al) whereas Rome IV criteria has not been validated for Turkish population yet. Threshold values of Rome IV criteria are based on Western data and language issues are important; if it is not translated properly, it may lead to misunderstanding. The different results are due to the use of Rome IV criteria, which is criticized for reliability in non-Western population in many articles $(25,26)$. Among other studies on IBS and skin diseases relation, Shalom et al. showed that IBS increased in patients with chronic urticaria (27). İslamoğlu et al. Reported that IBS was more common in atopic dermatitis group. In the study of Kim et al., a relationship was found between rosacea and IBS, while an increase in acne rosacea was found in cases with $\operatorname{IBS}(28)$.

There are limited number of studies investigating the relationship between acne and physical activity. Alshammrie et al. did not find a significant relationship between physical exercise and acne(29). Similarly, in another study conducted in Tehran, it was reported that there was no relationship between physical activity and acne(30). In our study, in accordance with the literature, no difference was found between the acne cases and the control group in terms of physical activity.

In recent studies, the role of a low glycemic index high fiber diet in the course of acne vulgaris treatment has been shown (31). In the study conducted by Kaufmann et al., a significant improvement was observed in the skin in the study in which he consumed $30 \mathrm{~g}$ of high-fiber breakfast cereal per day (32). Smith et al. demonstrated the positive effect of a diet rich in fiber and low glycemic index diet(33). In our study, we could not find a relationship between fiber consumption and acne. Further studies are needed to evaluate both fiber and glycemic index impact in large case series.

In a study investigating acne vulgaris and BMI, it was found that the incidence of severe acne was increased in adolescents with high BMI compared to normal ones(34). Inflammatory acne lesions were increased in patients with higher BMI and non-inflammatory acne lesions were found to be increased in those with low BMI(34). High BMI also triggers high levels of insulin-like growth factor-1 (IGF-1) release. IGF-1 stimulates keratinocyte proliferation, sebaceous lipogenesis, and androgen synthesis (34). In our study, no statistical difference was found between the acne cases and the control group in terms of BMI.

Smoking is related with many adverse effects on the skin(35). Smoking affects sebum production, induce microcirculation and oxidative stress(36). According to a study by Schäfer et al. acne prevalence was significantly higher in active smokers like our study (37). Smoking can increase vascular endothelial inflammation, causing exacerbation of both acne lesions and irritable bowel syndrome (38). Contrary to expectations, there was no relation between smoking and irritable bowel syndrome in current study.

Coffee consumption reduces non-melanoma skin cancer, melanoma, and rosacea (39). Consumption of foods containing a high glycemic index negatively affects acne, but there is no information in literature about coffee interaction. In our study, acne patients were consuming more coffee. Coffee type was not questioned; it would be informative to make further studies with different coffee types. Older people consume mostly Turkish coffee and young people increasingly consume filter coffee or instant coffee in Turkey.

A limitation of this study is the relatively small sample size.

\section{CONCLUSION}

This is the first study in the literature questioning bowel habits, physical activity, dietary fiber intake, and IBS all together in acne vulgaris patients. It is striking that coffee consumption is related with acne vulgaris. Although a significant relation between acne vulgaris and bowel habits or nutritional characteristics cannot be demonstrated, further large sample sized studies are needed on this subject, as there is strong evidence about brain-gut-skin axis existence.

There is no conflict interest.

There is no financial disclosure.

The data that support the findings of this study are available from the corresponding author 
upon reasonable reques

\section{REFERENCES}

1. Hazarika N, Rajaprabha RK. Assessment of Life Quality Index Among Patients with Acne Vulgaris in a Suburban Population. Indian J Dermatol . 2016;61(2):163-168.

2. Gollnick HP, Zouboulis CC. Not all acne is acne vulgaris. Dtsch Arztebl Int . 2014;111(17):301-312.

3. Stokes, JH, Pillsbury DM.The effect on the skin of emotional and nervous states: iII. Theoretical and practical consideration of a gastro-intestinal mechanism.Arch Derm Syphilol. 1930;22(6):962-993.

4. Kennedy PJ, Cryan JF, Quigley EM, Dinan TG, Clarke G. A sustained hypothalamic-pituitary-adrenal axis response to acute psychosocial stress in irritable bowel syndrome. Psychol Med . 2014;44(14):3123-3134.

5. Sweetser S, Camilleri M, Linker Nord SJ, et al. Do corticotropin releasing factor-1 receptors influence colonic transit and bowel function in women with irritable bowel syndrome?. Am J Physiol Gastrointest Liver Physiol . 2009;296(6):G1299-G1306

6. Mearin F, Lacy BE, Chang L, et al. Bowel Disorders [published online ahead of print, 2016 Feb 18]. Gastroenterology . 2016;S0016-5085(16)00222-5.

7. Holtmann GJ, Ford AC, Talley NJ. Pathophysiology of irritable bowel syndrome. Lancet Gastroenterol Hepatol . 2016;1(2):133-146

8. Doshi A, Zaheer A, Stiller MJ. A comparison of current acne grading systems and proposal of a novel system. Int J Dermatol . 1997;36(6):416-418.

9. Aydemir Ö, Guvenir T, Kuey L, Kultur S. Validity and reliability of Turkish version of hospital anxiety and depression scale. Turk Psikiyatri Derg isi 1997;8 (4):280-7.

10. Guiné RP, Duarte J, Ferreira M, et al. Knowledge about dietary fibres (KADF): development and validation of an evaluation instrument through structural equation modelling (SEM). Public Health . 2016;138:108118.

11. Ozgürsoy Uran BN, Vardar R, Karadakovan A, Bor S. The Turkish version of the Rome III criteria for IBS is valid and reliable. Turk J Gastroenterol . 2014;25(4):386-392

12 Drossman DA, Dumitrascu DL. Rome III: New standard for functional gastrointestinal disorders. $J$ Gastrointestin Liver Dis . 2006;15(3):237-241.

13. Lee PH, Macfarlane DJ, Lam TH, Stewart SM. Validity of the International Physical Activity Questionnaire Short Form (IPAQ-SF): a systematic review. Int J Behav Nutr Phys Act . 2011;8:115.

14. Samuels DV, Rosenthal R, Lin R, Chaudhari S, Natsuaki MN. Acne vulgaris and risk of depression and anxiety: A meta-analytic review. J Am Acad Dermatol . 2020;83(2):532-541

15 Bowe W, Patel NB, Logan AC. Acne vulgaris, probiotics and the gut-brain-skin axis: from anecdote to translational medicine. Benef Microbes . 2014;5(2):185-199.

16 Arck P, Handjiski B, Hagen E, et al. Is there a 'gut-brain-skin axis'?. Exp Dermatol . 2010;19(5):401-405.

17 Bercik P, Collins SM. The effects of inflammation, infection and antibiotics on the microbiota-gut-brain axis. Adv Exp Med Biol . 2014;817:279-289.

18 Rokowska-Waluch A, Pawlaczyk M, Cybulski M, et al. Stressful Events and Serum Concentration of Substance P in Acne Patients. Ann Dermatol . 2016;28(4):464-469.

19 Ford AC, Lacy BE, Talley NJ. Irritable Bowel Syndrome. N Engl J Med . 2017;376(26):2566-2578.

20 Barbara G, Cremon C, Annese V, et al. Randomised controlled trial of mesalazine in IBS. Gut . 2016;65(1):82-90. 
21. Choghakhori R, Abbasnezhad A, Hasanvand A, Amani R. Inflammatory cytokines and oxidative stress biomarkers in irritable bowel syndrome: Association with digestive symptoms and quality of life. Cytokine . 2017;93:34-43.

22. Addolorato G, Mirijello A, D'Angelo C, et al. State and trait anxiety and depression in patients affected by gastrointestinal diseases: psychometric evaluation of 1641 patients referred to an internal medicine outpatient setting. Int J Clin Pract . 2008;62(7):1063-1069.

23. Pimentel M, Chow EJ, Lin HC. Eradication of small intestinal bacterial overgrowth reduces symptoms of irritable bowel syndrome. Am J Gastroenterol . 2000;95(12):3503-3506.

24 Demirbaş A, Elmas ÖF. The relationship between acne vulgaris and irritable bowel syndrome: A preliminary study [published online ahead of print, 2020 May 28]. J Cosmet Dermatol . 2020;10.1111/jocd.13481.

25 Bai T, Xia J, Jiang Y, et al. Comparison of the Rome IV and Rome III criteria for IBS diagnosis: A cross-sectional survey. J Gastroenterol Hepatol . 2017;32(5):1018-1025.

26 Ghoshal UC. Pros and Cons While Looking Through an Asian Window on the Rome IV Criteria for Irritable Bowel Syndrome: Pros. J Neurogastroenterol Motil . 2017;23(3):334-340.

27 Shalom G, Magen E, Babaev M, et al. Chronic urticaria and irritable bowel syndrome: a cross-sectional study of 11271 patients. Br J Dermatol . 2018;178(3):e204-e206.

28 Kim M, Choi KH, Hwang SW, Lee YB, Park HJ, Bae JM. Inflammatory bowel disease is associated with an increased risk of inflammatory skin diseases: A population-based cross-sectional study. $J$ Am Acad Dermatol . 2017;76(1):40-48.

29 Alshammrie FF, Alshammari R, Alharbi RM, Khan FH, Alshammari SK. Epidemiology of Acne Vulgaris and Its Association With Lifestyle Among Adolescents and Young Adults in Hail, Kingdom of Saudi Arabia: A Community-Based Study. Cureus . 2020;12(7):e9277

30 Ghodsi SZ, Orawa H, Zouboulis CC. Prevalence, severity, and severity risk factors of acne in high school pupils: a community-based study. J Invest Dermatol . 2009;129(9):2136-2141.

31 Kucharska A, Szmurło A, Sińska B. Significance of diet in treated and untreated acne vulgaris. Postepy Dermatol Alergol . 2016;33(2):81-86

32 Kaufman WH. The diet and acne. Arch Dermatol . 1983;119(4):276.

33 Smith RN, Mann NJ, Braue A, Mäkeläinen H, Varigos GA. A low-glycemic-load diet improves symptoms in acne vulgaris patients: a randomized controlled trial. Am J Clin Nutr . 2007;86(1):107-115.

34 Sas K, Reich A. High Body Mass Index is a Risk Factor for Acne Severity in Adolescents: A Preliminary Report. Acta Dermatovenerol Croat . 2019;27(2):81-85.

35 Firooz A, Sarhangnejad R, Davoudi SM, Nassiri-Kashani M. Acne and smoking: is there a relationship?. BMC Dermatol . 2005;5:2.

36 Capitanio B, Sinagra JL, Ottaviani M, Bordignon V, Amantea A, Picardo M. Acne and smoking. Dermatoendocrinol . 2009;1(3):129-135.

37 Schäfer T, Nienhaus A, Vieluf D, Berger J, Ring J. Epidemiology of acne in the general population: the risk of smoking. Br J Dermatol . 2001;145(1):100-104

38 Helvaci M. R, Ayyildiz O, Algin M.C., Aydin Y, Abyad A,Pocock, L. Irritable bowel syndrome and smoking. World Family Medicine Journal: Incorporating the Middle East Journal of Family Medicine . 2017; 99 (5588): 1-5.

39 Bray ER, Kirsner RS, Issa NT. Coffee and skin-Considerations beyond the caffeine perspective. $J$ Am Acad Dermatol . 2020;82(2):e63. 
Table 1 . Socio-demographic characteristics of the participants

\begin{tabular}{lllllll}
\hline & Patient & Patient & Control & Control & $\mathbf{\chi}^{\mathbf{2}}$ & $\mathbf{p}$ \\
\hline & $\mathrm{n}$ & $\%$ & $\mathrm{n}$ & $\%$ & & \\
Gender & & & & & & \\
Female & 85 & 83,3 & 76 & 73,1 & 2,601 & 0,107 \\
Male & 17 & 16,7 & 28 & 26,9 & & \\
Education level & & & & & & \\
Primary school & 2 & 2,0 & 4 & 3,8 & 5,878 & 0,118 \\
Secondary school & 6 & 5,9 & 14 & 13,5 & & \\
High school & 34 & 33,3 & 39 & 37,5 & & \\
University / college & 60 & 58,8 & 47 & 45,2 & & \\
Economic situation & & & & & & \\
Income more than expenses & 25 & 24,5 & 7 & 6,7 & 22,287 & $\mathbf{0 , 0 0 0}$ \\
Income equal to expenses & 53 & 52,5 & 85 & 81,8 & & \\
Income less than expenses & 24 & 23,5 & 12 & 11,5 & & \\
Family type & & & & & & \\
Large family & 43 & 42,2 & 20 & 19,2 & 32,944 & $\mathbf{0 , 0 0 0}$ \\
Nuclear family & 36 & 35,3 & 70 & 67,3 & & \\
Alone & 23 & 22,5 & 14 & 13,5 & & \\
Smoking & & & & & & \\
No & 81 & 79,4 & 69 & 66,3 & 4,441 & $\mathbf{0 , 0 3 5}$ \\
Yes & 21 & 20,6 & 35 & 33,7 & & \\
\hline
\end{tabular}

Table 2: The nutritional and bowel habits of the participants

\begin{tabular}{lllllll}
\hline & Patient & Patient & Control & Control & $\boldsymbol{\chi}^{\mathbf{2}}$ & $\mathbf{p}$ \\
\hline & $\mathbf{n}$ & $\mathbf{9}$ & $\mathbf{n}$ & $\mathbf{\%}$ & & \\
Breakfast & & & & & & \\
Do not have & 9 & 8,8 & 6 & 5,8 & 4,972 & 0,174 \\
At home & 74 & 72,5 & 85 & 81,7 & & \\
Outside & 19 & 18,7 & 13 & 12,5 & & \\
Lunch & & & & & & \\
Not eating & 22 & 21,6 & 25 & 24,0 & 4,426 & 0,219 \\
At home & 34 & 33,3 & 35 & 33,7 & & \\
Outside & 38 & 37,3 & 28 & 26,9 & & \\
At workplace & 8 & 7,8 & 16 & 15,4 & & \\
Dinner & & & & & & \\
At home & 89 & 87,3 & 98 & 94,2 & 3,975 & 0,264 \\
Outside & 13 & 12,7 & 6 & 5,8 & & \\
& $\mathbf{M e a n}$ & $\mathbf{S D}$ & $\mathbf{M e a n}$ & $\mathbf{S D}$ & $\mathbf{t}$ & $\mathbf{p}$ \\
The amount of water consumed daily (glass) & 6,69 & 4,21 & 6,93 & 4,25 & 0,401 & 0,689 \\
The amount of tea consumed per day (tea cup) & 3,68 & 3,06 & 2,94 & 3,68 & 1,575 & 0,117 \\
The amount of coffee consumed per day (coffee cup) & 1,44 & 1,90 & 0,98 & 1,22 & $-2,065$ & $\mathbf{0 , 0 4}$ \\
Number of vegetable/salad containing meals per week & 4,50 & 3,67 & 4,16 & 2,72 & 0,770 & 0,442 \\
Number of fruits per week & 6,40 & 4,96 & 5,82 & 3,80 & 0,935 & 0,351 \\
Amount of fast-food eaten per week & 2,07 & 2,19 & 2,03 & 2,59 & 0,119 & 0,905 \\
Number of defecations per week & 6,99 & 4,49 & 6,62 & 4,88 & 0,558 & 0,577 \\
\hline
\end{tabular}


Table 3 . Anxiety-depression and dietary fiber knowledge scores of the participants

\begin{tabular}{lllll}
\hline & Patient & Control & & \\
& Mean \pm SD & Mean \pm SD & t & p \\
\hline KADF $^{+}$ & $72,80 \pm 7,87$ & $71,85 \pm 8,38$ & 0,836 & 0.404 \\
$\mathbf{H D S}^{+}+$ & $9,01 \pm 2,82$ & $9,46 \pm 2,04$ & 1,320 & 0.188 \\
HAS $^{++}+$ & $10,48 \pm 3,08$ & $9,12 \pm 3,71$ & 2,848 & $\mathbf{0 . 0 0 5}$ \\
\hline
\end{tabular}

$+\mathrm{KADF}:$ Knowledge about dietary fiber

++ HDS : Hospital Depression Scale

+++ HAS : Hospital Anxiety- Scale

Table 4 . Relationship between the global acne grading scores of the cases and the presence of IBS

\begin{tabular}{llllll}
\hline & & GAGS + & GAGS + & GAGS + & P \\
\hline \multirow{4}{*}{ IBS } & & Mild n $(\%)$ & Medium n $(\%)$ & Severe/Very Severe n (\%) & \\
& Present & $37(43,0)$ & $45(52,3)$ & $4(4,7)$ & 0,162 \\
Total & Not present & $3(18,8)$ & $12(75,0)$ & $1(6,3)$ & \\
\hline
\end{tabular}

Table 5 . Correlations between Global acne grading scores and Physical Activity Scores, BMI and dietary fiber knowledge of the subjects

\begin{tabular}{lllll}
\hline & KADF + & PAS ++ & GAGS +++ & BMI ++++ \\
\hline KADF r p & 1 & & & \\
PAS r p & $-0,500,615$ & 1 & & \\
GAS r p & $0,1700,088$ & $0,1420,154$ & 1 & \\
\hline
\end{tabular}

+ KADF : Knowledge About Dietary Fiber

++ PAS : Physical Activity Scores

+++ GAGS : Global Acne Grading Scores

++++ BMI : Body Mass Index 